\title{
Mini-symposium
}

\section{Free radicals and redox signalling in cardiovascular disease: introduction}

$\mathrm{R}$

eactive oxygen species (ROS) are highly reactive chemical species comprising both free radicals such as superoxide and non-radicals such as hydrogen peroxide. When the normal balance between ROS generation and antioxidant systems is perturbed, a state of oxidative stress is said to exist, which has traditionally been considered deleterious due to tissue oxidation and damage. Recently, however, ROS have been recognised to exert more subtle effects. Tightly regulated ROS production modulates intracellular signalling pathways ("redox signalling") and can induce highly specific changes in cell phenotype, especially in pathological settings. ROS also inactivate the signalling molecule nitric oxide (NO) and cause endothelial dysfunction, which may itself be a contributor to disease pathogenesis.

The following articles in this minisymposium address several topical aspects of the roles of ROS in cardiovascular disease. The overview article by Shah and Channon considers general mechanisms, effects, and relevance of redox signalling, and is followed by an article by Jin and Berk addressing recently identified novel redox signalling mechanisms. Kathy Griendling reviews the fascinating family of enzymes known as NADPH oxidases, which have recently been identified as major players in redox signalling in several cardiovascular disorders. Finally, Verhaar and colleagues discuss the potential pathogenic importance of superoxide production by NOS, the enzyme that normally generates NO but can switch to ROS production when the NOS co-factor tetrahydrobiopterin is deficient-for example, in diabetic vasculopathy. We hope that this minisymposium will provide an up-to-date review of the important field of oxidative stress and redox signalling and its relevance to clinical cardiovascular disease.

A M Shah
Department of Cardiology, Guy's King's \& St
Thomas's School of Medicine, King's College
London, UK; ajay.shah@kcl.ac.uk
K M Channon
Department of Cardiovascular Medicine,
University of Oxford, UK

\section{IMAGES IN CARDIOLOGY}

\section{Oversized semi-compliant balloon to dilate an "undilatable" stenosis}

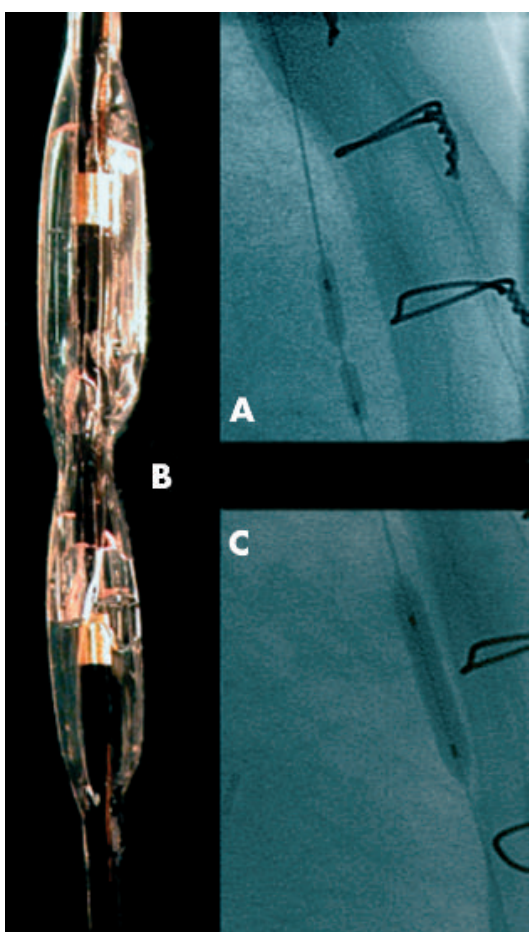

\begin{abstract}
56 year old woman presented for elective percutaneous coronary intervention (PCI) following the recurrence of limiting angina two years after coronary bypass operation. In this short time, the saphenous vein graft to her right coronary artery had blocked, and the left internal mammary artery (LIMA) anastomosed to her left anterior descending artery (LAD) showed a long, severe, heavily calcified stenosis proximal to the anastomosis. As the native vessels were not amenable to PCI, a decision was made to tackle the LIMA-LAD lesion.

The lesion was crossed with a BMW guidewire and balloon pre-dilatation attempted. However, multiple coronary balloons (diameters $2.0-3.0 \mathrm{~mm}$ ) failed to crack the severely calcified stenosis (panel A) Two balloons ruptured (inflation pressures being more than $20 \mathrm{~atm}$ ), leading to localised coronary dissection. Usage of cutting balloon (Boston Scientific), which includes microsurgical blades to incise the calcified plaque, was attempted, but even high inflation pressures failed to impact on the narrowing. One of the ruptured balloons (Boston Scientific Maverick $2.0 \mathrm{~mm}$ balloon, inflated at $22 \mathrm{~atm}$ ), retained the circumferential shape of the stenosis even after removal and washing (panel B). Eventually, an oversized 3.5 semi-compliant balloon (Medtronic Extensor $3.5 \mathrm{~mm}$ ) succeeded in dilating the stenosis (panel C), that was then successfully treated with a drug eluting stent.

The treatment of non-dilatable, severely calcified stenosis remains challenging. The use of rotational ablation techniques (for example, Rotablator) may solve the problem, but vessel tortuosity may limit their utility in distally located stenoses. In this setting, using an oversized semi-compliant or a non-compliant balloon, with careful step-by-step inflations, may be
\end{abstract} helpful by permitting an increase of radial dilation force. 